\title{
Influence of model parameters on a simulation of x-ray irradiated materials: example of XTANT code
}

\author{
Nikita Medvedev ${ }^{* a, b}$, Vladimir Lipp ${ }^{c}$ \\ ${ }^{a}$ Institute of Physics, Academy of Science of Czech Republic, Na Slovance 1999/2, 18221 Prague 8, Czech Republic \\ ${ }^{\mathrm{b}}$ Institute of Plasma Physics, Academy of Science of Czech Republic, Za Slovankou 1782/3, 18200 Prague 8, Czech \\ Republic \\ ${ }^{c}$ Center for Free-Electron Laser Science, DESY, D-22607 Hamburg, Germany
}

\begin{abstract}
In this contribution an analysis of influence of model parameters on the results of simulations of material properties under free-electron laser irradiation is presented. It is based on the in-house hybrid code XTANT (X-ray-induced Thermal And Nonthermal Transition; N. Medvedev et. al, Phys. Rev. B 91 (2015) 054113), which combines tight binding molecular dynamics for atoms with Monte Carlo treatment of high-energy electrons and core-holes, and Boltzmann collision integrals for nonadiabatic (electron-phonon) coupling. Different parameterizations of transferable tight binding method for silicon are analyzed, namely basis sets $\mathrm{sp}^{3}$ and $\mathrm{sp}^{3} \mathrm{~s}^{*}$. The $\mathrm{sp}^{3}$ parameterization seems to provide a better agreement of the silicon damage threshold with experimental data. Further, the influence of different schemes of molecular dynamics periodic boundaries simulation is compared: constant volume vs Parrinello-Rahman constant pressure. Constant-volume scheme gives a better agreement with experimental transient properties, as could be expected. Parameters entering the calculations of optical properties are analyzed, showing virtually no effect on the outcome beyond trivial broadening of the peaks of the optical coefficients.
\end{abstract}

Keywords: Transferable tight binding, Silicon, Free-electron laser, XTANT

\section{INTRODUCTION}

Development of the $4^{\text {th }}$ generation intense free-electron lasers (FELs) rendered it possible to create and use extreme ultraviolet and soft X-ray radiation as ultrashort pulses of intensities inaccessible before ${ }^{1-3}$. FEL pulses can induce phase transitions in materials, changing their structure and electronic properties via a sequence of transient processes ${ }^{4,5}$. First, photo-electrons, excited by a laser pulse, produce electron cascades, exciting secondary free-electrons and creating a nonequilibrium electronic state which typically lasts for a few tens of femtoseconds ${ }^{6-8}$. Simultaneously, Auger-decays of core holes may occur in case if the photon energy is sufficient for their excitation. This process typically also requires a few femtoseconds $(<10 \mathrm{fs})^{9}$. Then, electronic excitation leads to modification of the interatomic potential energy surface. It leads to nonthermal atomic kinetics, such as nonthermal melting. It manifests on a scale of a few hundreds of femtoseconds (in silicon) ${ }^{10}$. Later, on picosecond timescales, electron-phonon (or more general electron-ion) coupling heats up the lattice and may lead to thermal melting ${ }^{10}$. The two processes, thermal and nonthermal, can also intertwine with each other ${ }^{10}$.

One of the most important advantages of femtosecond pulses is that the pulse duration is comparable with characteristic timescales of the processes in solids, e.g. the typical timescales of electron cascades in material, including its nonequilibrium stage and phase transitions ${ }^{11}$. Therefore, it allows testing theoretical models against experimental data with a sufficient time-resolution. So far, most of the femtosecond time-resolved experimental schemes used optical probe pulses, therefore there is a need for a theoretical connection between the optical properties and underlying (atomic and electronic) structure evolution ${ }^{12}$.

In this respect, comparison of model calculations to experimental data unavoidably goes through the stage of connecting the calculated data to the observable optical coefficients ${ }^{13}$. In our previously published model ${ }^{12}$, such a connection was

*Email: nikita.medvedev@fzu.cz

Damage to VUV, EUV, and X-ray Optics VI, edited by Libor Juha, Saša Bajt, Regina Soufli, Proc. of SPIE Vol. 10236, 102360I · C 2017 SPIE · CCC code: 0277-786X/17/\$18 · doi: 10.1117/12.2266953 
implemented using a complex dielectric function approach. Thus, this report explores how the theoretical uncertainty in the model parameters influences the results of such calculations. We evaluate which sets of parameters and which numerical schemes are more appropriate for modeling of femtosecond FEL-pulse irradiation of solids. This analysis is based on the recently developed code XTANT, which will be briefly described in the next section.

\section{OVERVIEW OF XTANT CODE}

The developed hybrid code XTANT ${ }^{14}$ (which stands for $\underline{X}$-ray-induced Thermal $\underline{\text { And }}$ Nonthermal $\underline{T r a n s i t i o n s}^{10}$ ), consists of the following largely independent modules:

(a) Monte Carlo (MC) module that follows nonequilibrium high-energy electron and core-hole kinetics after photoabsorption. It is based on the so-called asymptotic trajectory Monte Carlo scheme ${ }^{15}$. The cross sections of inelastic scattering used are based on the complex-dielectric function formalism as developed by Ritchie and Howie ${ }^{16}$. The appropriate cross sections for silicon, that will be used throughout this paper, were presented in Ref.[17] and its approximations were already analyzed in Ref.[18].

(b) The module tracing the electron distribution of valence and low-energy conduction-band electrons relies on the Boltzmann equation for homogeneous systems:

$$
\frac{d f_{e}\left(E_{i}\right)}{d t}=I_{e-e}+I_{e-i}
$$

where $f_{e}\left(E_{i}\right)$ is the transient electron distribution (a fractional occupation number on the energy level $E_{i}$ ); $I_{e-e}$ is the electron-electron collision integral, and $I_{e-i}$ is the electron-ion scattering integral which reduces to electron-phonon scattering in the case of long timescales, a harmonic potential and an ideal crystal structure ${ }^{19}$. We assume an instantaneous electron thermalizaion (infinitely fast electron-electron scattering), which leads to Fermi-Dirac distribution. The coupling of electrons to ions, $I_{e-i}$, is calculated via the Boltzmann collision integral beyond the Fermi's Golden Rule as described in detail in Ref.[19].

Such a separation between the high-energy electron domain (modeled within MC) and low-energy electron domain (modeled with the Boltzmann equation) is possible due to the specific shape of the transient electron distribution under FEL irradiation called "bump on hot tail" distribution ${ }^{8,20,21}$. This transient distribution consists of nearly thermalized majority of low-energy electrons, and a long tail of high-energy electrons far from equilibrium. Hence, separate treatment of the two fractions with appropriate methods is justified.

(c) The transferable tight binding (TB) method is used to calculate the transient Hamiltonian, electron band structure, collective potential energy surface for atoms, and matrix elements used in the electron-ion coupling entering Bolztmann collision integral ${ }^{14,19}$. This method allows to include influence of the electron distribution, $f_{e}$, on the interatomic potential, $\Phi$ :

$$
\Phi\left(\left\{r_{j k}(t), t\right\}\right)=\sum_{i=1}^{N} f_{e}\left(E_{i}\right) E_{i}(t)+E_{r e p}\left(\left\{r_{j k}(t)\right\}\right),
$$

where energy levels are directly obtained by diagonalization of the tight binding Hamiltonain: $E_{i}(t)=\langle i(t)|\hat{H}(\{r(t)\})| i(t)\rangle$; and $E_{\text {rep }}$ is the core-core repulsive potential.

The tight binding method used in our earlier works on silicon, Refs.[19,22,23], was based on $\mathrm{sp}^{3}$ parameterization. It takes into account only the $3 s, 3 p_{x}, 3 p_{\mathrm{y}}$ and $3 p_{\mathrm{z}}$ valence orbitals of silicon. The TB parameters and the repulsive core-core energy parameters were taken from Ref.[24].

Additionally, we implemented an $\mathrm{sp}^{3} \mathrm{~s}^{*}$-basis parameterization, which adds an effective $\mathrm{s}^{*}$ level to the abovementioned valence orbitals of silicon in order to account for higher-lying orbitals. The parameters for $\mathrm{sp}^{3} \mathrm{~s}^{*} \mathrm{~TB}$ parameterization and corresponding repulsive term of the potential energy are taken from Ref.[25].

(d) Molecular Dynamics (MD) approach traces atomic trajectories of all atoms in the super-cell with periodic boundary conditions (PBC). XTANT includes two alternative modes for PBC: constant volume simulation (V=const, NVE ensemble), or constant pressure simulation ( $\mathrm{P}=$ const, NPH ensemble) modeled within Parrinello-Rahman scheme ${ }^{14,26}$. 
The case of $\mathrm{V}=$ const simulation corresponds to the solution of the standard Newtonian equations of motion for atoms, whereas $\mathrm{P}=$ const case includes additional variables in the Lagrangian related to the super-cell size and shape. In the Parrinello-Rahman method it results in the following equations of motion ${ }^{26,27}$ :

$$
\begin{aligned}
& \ddot{\mathbf{s}}_{i}=-\partial \Phi\left(\left\{r_{i j}(t), t\right\}\right) / d s_{i}\left(g^{-1} / M_{a t}\right)-g^{-1} \dot{g} \dot{\mathbf{s}}_{i} \\
& \ddot{h}_{\alpha \beta}=1 / M_{P R}\left(\sum_{i=1}^{N} M_{a t} \dot{\mathbf{s}}_{i}^{T}\left(h \dot{\mathbf{s}}_{i}\right)-P \partial \Omega / \partial h_{\alpha \beta}-\partial \Phi\left(\left\{r_{i j}(t), t\right\}\right) / \partial h_{\alpha \beta}\right),
\end{aligned}
$$

where $\boldsymbol{s}_{i}$ is the relative atomic coordinate within the super-cell; $\Phi\left(\left\{r_{i j}\right\}\right)$ is the potential energy surface defined by Eq.(2); $g=h^{T} h$, where $h$ is the (3x3) matrix spanning the MD super-cell; $M_{a t}$ is the mass of an atom; $M_{P R}=N_{a t} M_{a t} / 25.5$ is the chosen effective mass of the super-cell ${ }^{26}, N_{a t}$ is the number of atoms in the super-cell; $\Omega=\operatorname{det}(h)$ is the transient volume of the simulation box; $P$ is the external (atmospheric) pressure. We use Verlet algorithm in its velocity form with the timestep of $0.01 \mathrm{fs}$ for propagation of the atomic coordinates and velocities in time, as well as for the super-cell coordinates and velocities (in a case of NPH ensemble) ${ }^{28,29}$. This time-step is chosen as to achieve convergence in calculations of both, Molecular Dynamics, as well as the Boltzmann collision integral ${ }^{19}$. Additionally, a velocity scaling is used for transferring energy to atoms which was calculated from the Boltzmann integral, Eq.(1) in the module (b) above $^{19}$.

(e) The complex dielectric function (CDF), which is used to calculate optical properties, is modeled within the random phase approximation, $\mathrm{RPA}^{12}$. It does, however, account for transient band structure of the particular material without assuming free-electron-gas approximation, $\varepsilon(\omega)$ :

$$
\varepsilon^{\alpha \beta}(\omega)=\delta_{\alpha, \beta}+\frac{e^{2} \hbar^{2}}{m^{2} \Omega e_{0}} \sum_{i, j} \frac{F_{i, j}}{\left(\hbar \omega_{i, j}\right)^{2}} \frac{f_{e}\left(E_{i}\right)-f_{e}\left(E_{j}\right)}{\hbar \omega-\hbar \omega_{i, j}+i \hbar \gamma},
$$

where $e$ is the free-electron charge; $m$ is the mass of a free electron; $e_{0}$ is the vacuum permittivity in SI units, and $\hbar$ is the Planck's constant; $\hbar \omega_{i, j}=E_{j}-E_{i}$ is the transition energy between two eigenstates $|i\rangle$ and $|j\rangle ; f_{e}\left(E_{i}\right)$ and $f_{e}\left(E_{j}\right)$ are the corresponding transient occupation numbers (electron distribution function mentioned above); $\omega$ is the frequency as a variable in the complex dielectric function; $F_{i, j}=|\langle i|p| j\rangle|^{2}$ is the oscillator strength. It is obtained within the Trani's formalism for tight binding calculations ${ }^{30}$. The parameter $\gamma$ (sometimes referred to as a damping factor, a decay constant, or an inverse electron scattering time) entering here in the RPA approximation should tend to zero, however, is kept finite for numerical purposes. Its influence on the $\mathrm{CDF}$, among other things, will be discussed in the course of the paper.

\section{RESULTS AND DISCUSSIONS}

The results of modeling of silicon exposed to an FEL pulse with XTANT code presented below are divided into three subsections. In subsection 1, we analyze the dependence on the TB parameterization, namely, the above-mentioned $\mathrm{sp}^{3}$ basis $\operatorname{set}^{24}$ is compared with the $\mathrm{sp}^{3} \mathrm{~s}^{*}$ basis $\mathrm{set}^{25}$. Then, in subsection 2 we proceed to the analysis of the numerical parameter $\gamma$ entering Eq. (4) for optical parameters calculations within the RPA approximation for the CDF. After that, having proved the applicability of the parameters we use, in subsection 3 we apply the model to compare the NVE $(\mathrm{V}=$ const $)$ and $\mathrm{NPH}(\mathrm{P}=\mathrm{const})$ ensembles for simulation of the transient properties of materials under irradiation.

\section{TB basis set dependence}

XTANT calculations of the damage threshold in silicon are compared for the both basis sets: $\mathrm{sp}^{3}$ from Ref.[24] and $\mathrm{sp}^{3} \mathrm{~s}^{*}$ basis set from Ref.[25]. The calculated results are shown in Table I. The results show that the proposed $\mathrm{sp}^{3} \mathrm{~s}^{*}$ parameterization from Ref.[25] produces a stiffer potential than $\mathrm{sp}^{3}$ basis set from Ref.[24]. Such a stiffer potential makes material more resilient to radiation damage. It seems, it overestimates the damage threshold in comparison to experimental data $(\sim 0.88 \mathrm{eV} / \mathrm{atom})^{31}$. Thus, further we use the $\mathrm{sp}^{3}$ parameterization which produces the damage threshold closer to experimental data. 
Table I. Damage threshold parameters for non-thermal (with thermal contribution ${ }^{22}$ ) melting of silicon.

\begin{tabular}{|c|c|c|c|}
\hline & Dose, (eV/atom) & $\begin{array}{c}\text { Electron density, } \\
(\% \text { of valence electrons })\end{array}$ & Electron temperature, (K) \\
\hline $\mathrm{sp}^{3}$ basis set & 24 & 5 & 11000 \\
\hline $\mathrm{sp}^{3} \mathrm{~s}^{*}$ basis set & 0.9 & 14 & 20000 \\
\hline
\end{tabular}

\section{Model parameters in calculation of optical properties}

Using the $\mathrm{sp}^{3} \mathrm{~TB}$ parameterization, we proceed with calculations of the optical properties. They are calculated via the linear response theory within RPA approximation, Eq. (4) ${ }^{12}$. The parameter $\gamma$ entering Eq. (4) in our former works was set to be $\gamma=1.510^{13} \mathrm{~s}^{-1}$. Here we show what difference a choice of this parameter makes. For that, we calculated optical coefficients: refractive indices $n$ and extinction coefficients $k$ of the equilibrium silicon, Figure 1. This figure shows calculations for the $\Gamma$-point, which produces strong spikes at selected energies.

Figure 1 demonstrates that, as claimed in Ref.[12], the parameter $\gamma$ does not affect the results beyond the broadening of the peaks in CDF. Values of $\gamma$ below $10^{14} \mathrm{~s}^{-1}$ lead to almost identical peaks in the optical coefficients, whereas for larger values of $\gamma$ the peaks start to overlap creating much smoother overall curves. Note that these calculations were performed for 64 atoms in the super-cell. Increasing the number of atoms also slowly smoothens the CDF as shown in Ref.[12].
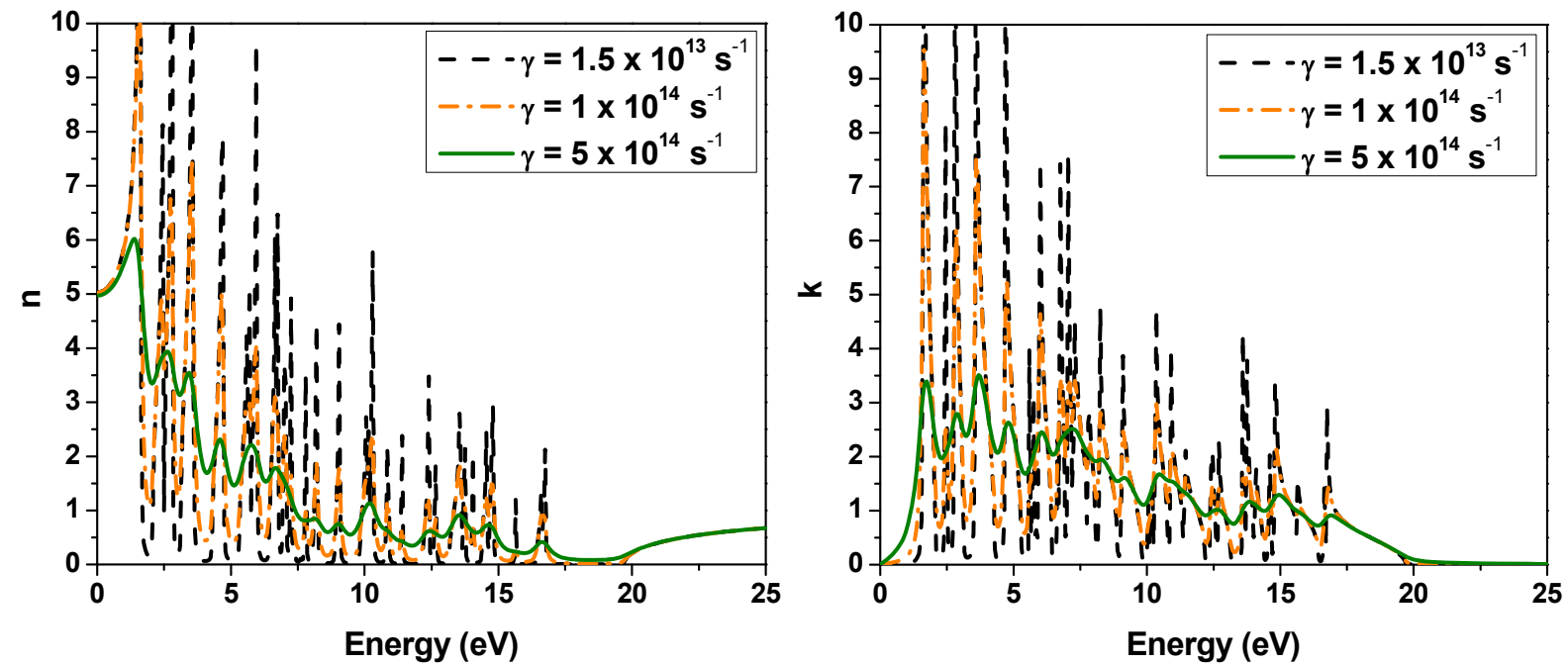

Figure 1. Optical $\mathrm{n}$ (left panel) and $\mathrm{k}$ (right panel) parameters of silicon calculated at the $\Gamma$-point for different values of the parameter $\gamma$, Eq. (4).

Another way to smoothen the curves is to increase the number of k-points (grid in the reciprocal space) ${ }^{12}$. Figure 2 shows the calculated CDF for $2197(13 \times 13 \times 13)$ k-points. In this case, a change of $\gamma$ below $10^{14} \mathrm{~s}^{-1}$ does not have almost any observable effect on the results, and only increasing it above this value starts to noticeably smoothen peaks. Thus, we can conclude that the choice of the $\gamma$ parameter has only a trivial influence on the outcome by smoothing the curves, and further we can use previously applied value of $\gamma=1.5 \times 10^{13} \mathrm{~s}^{-1}$. 

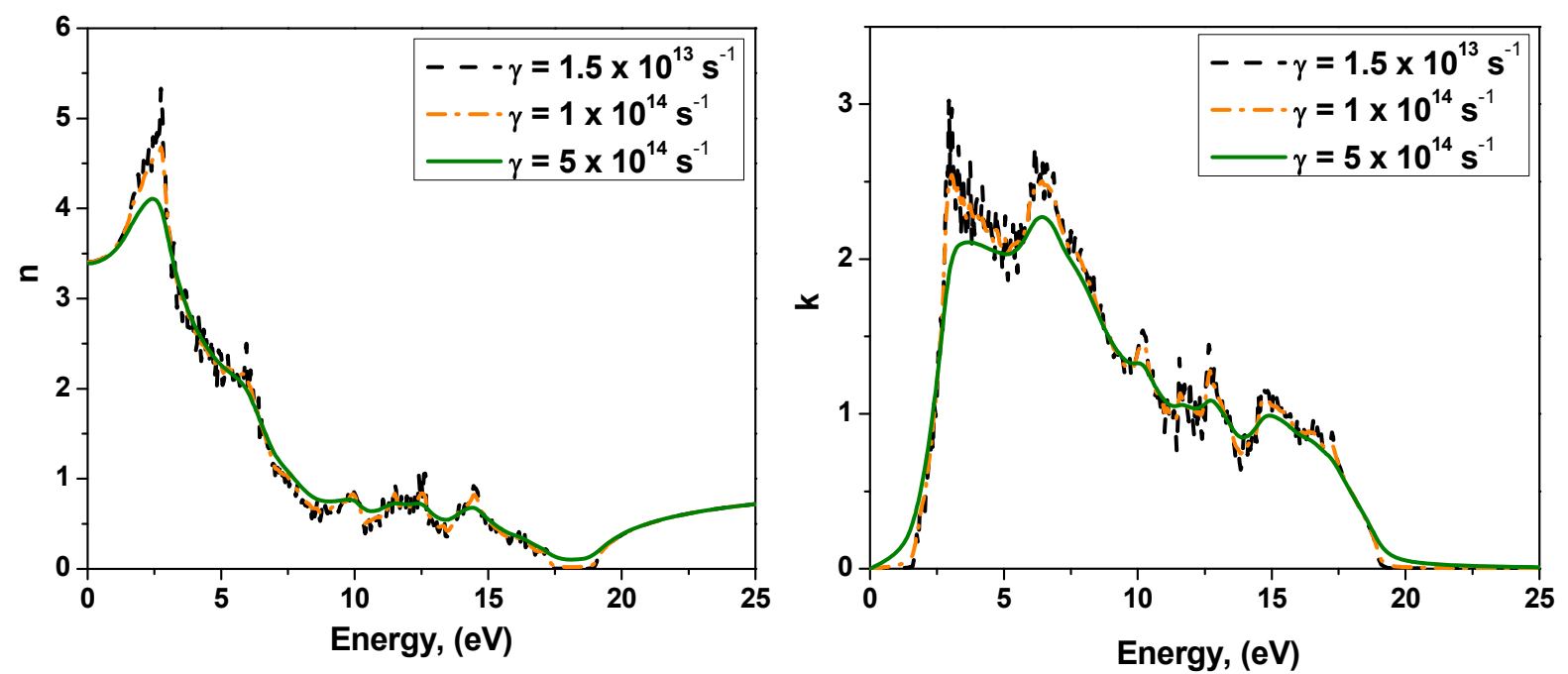

Figure 2. Optical n (left panel) and k (right panel) parameters of silicon calculated for 13x13x13 k-points for different values of parameter $\gamma$, Eq. (4).

\section{Constant volume vs constant pressure simulations}

To investigate the difference between a constant volume simulation (NVE thermodynamic ensemble) and a constant pressure simulation (NPH thermodynamic ensemble), we run XTANT calculations for the following parameters: silicon irradiated with FEL pulse of $30 \mathrm{eV}$ photon energy, 60 fs duration FWHM, 1 eV/atom absorbed dose (216 atoms in the simulation box, $0.01 \mathrm{fs}$ time step). We simulate the probe pulse reflectivity at $625 \mathrm{~nm}$ probe wavelength under $70.5^{\circ}$ incidence; for details on the angular dependence of the optical coefficients see e.g. Eq.(1) in Ref. [12]. The calculated probe reflectivity is shown in Figure 3.

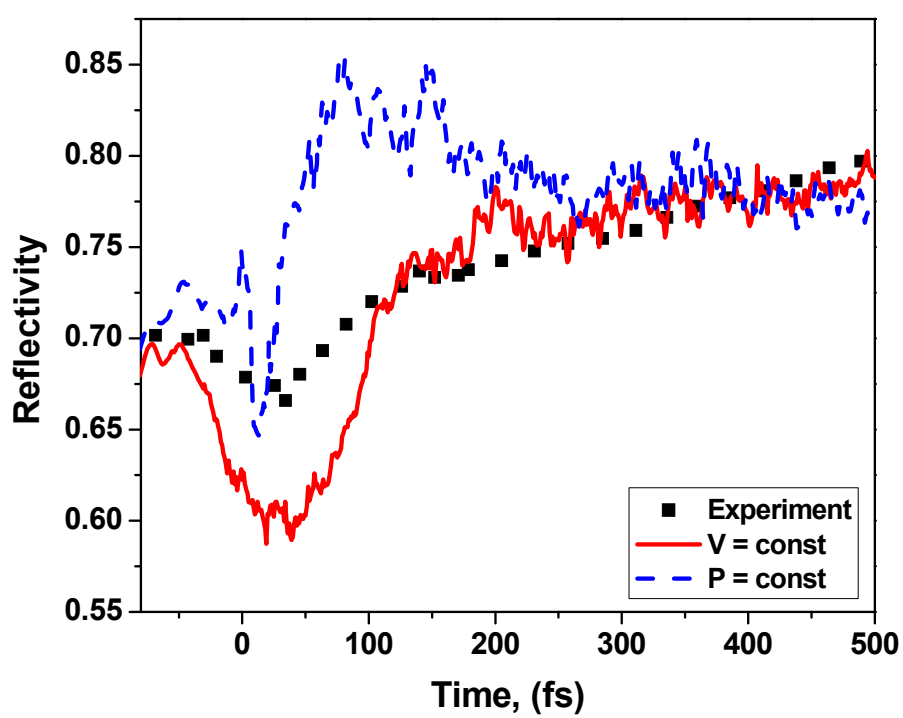

Figure 3. Optical reflectivity in silicon irradiated with absorbed dose $\sim 1 \mathrm{eV} /$ atom, $60 \mathrm{fs}$ laser pulse, modelled photon energy of $30 \mathrm{eV}$. Two modeling cases are compared: constant volume $(\mathrm{V}=$ const $)$ vs constant pressure $(\mathrm{P}=$ const $)$ simulation. Both are compared with experimental data from Ref.[32]. 
For the case of the constant volume simulation (see the red solid line in Fig. 3), we see the decay in the reflectivity during the laser pulse (within a few tens of femtoseconds), in a good agreement with the experimental points (black squares). This decay is caused by the X-ray-induced increase in the electron density ${ }^{12}$. Simultaneously, the band gap of silicon is shrinking due to ongoing nonthermal melting, which completes within $\sim 300-500 \mathrm{fs}^{22}$. As a result of the band gap shrinkage, the reflectivity increases, showing the transient metallic state.

In contrast, constant pressure simulation produces drastically different dynamics. For our chosen simulation parameters, the NPH ensemble gives unphysically quick collective reaction of the super-cell, Figure 4. Too fast expansion of the super-cell leads to rise of the optical reflectivity in Figure 3. The timescale of this rise does not correspond to what the experiment shows.

Later, the reflectivity relaxes to the value close to the experimental one, but approaching it from a wrong side: it decreases from top, whereas experimental value rises up from bottom. Thus, the results demonstrate that the constant volume simulations are closer to the experimental data than constant pressure simulations. A better performance of the constant-volume simulation could be expected at sub-picosecond timescales, which are shorter than typical timescales of physical expansion of solids.

The constant pressure simulations are a valuable modeling technique which allows to treat phase transitions and to distinguish between different phases produced. For example, only the use of NPH ensemble allowed us to describe formation of low-density liquid and high-density liquid phases of silicon under FEL irradiation in Ref.[22]. However, our present results demonstrate that such simulations must be interpreted with caution. They may lead to wrong transient dynamics and characteristic timescales, even though the final state of the material may be reproduced correctly. It is especially noticeable for small super-cell sizes, whereas for large numbers of atoms the simulation results approach those of the constant volume simulations.

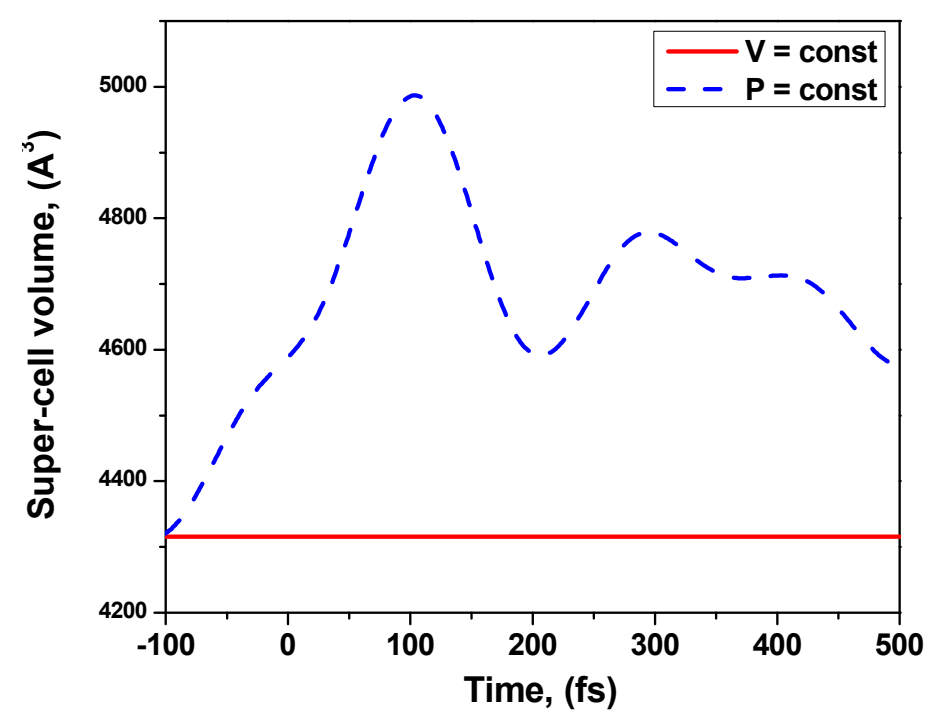

Figure 4. Volume of silicon super-cell irradiated with the absorbed dose of $1 \mathrm{eV} / \mathrm{atom}, 60 \mathrm{fs}$ laser pulse, modelled photon energy of $30 \mathrm{eV}$. Two modeling cases are compared: $\mathrm{V}=$ const vs $\mathrm{P}=$ const.

\section{CONCLUSIONS}

In this work we analyzed the influence of the simulation parameters on the physical results of the hybrid code XTANT. We demonstrated that the tight binding parameterization based on the $\mathrm{sp}^{3}$ basis set from Ref.[24] produces damage threshold closer to the experimental value than the $\mathrm{sp}^{3} \mathrm{~s}^{*}$ basis set from Ref.[25]. Further, we showed that the parameter $\gamma$ 
in the RPA approximation of the complex dielectric function only broadens/narrows the peaks for $\Gamma$-point calculation, and is almost unnoticeable for multiple-k-points calculations. Last, we showed that, for the considered case of nonthermal melting, the NVE ensemble (constant volume) calculations show a better agreement with the experimental results for the transient behavior of irradiated silicon than the NPH ensemble (constant pressure, calculated within the Parrinello-Rahman scheme). This result indicates that one must exercise caution applying constant pressure simulations to transient dynamical properties (as opposed to the description of the equilibrium state). In summary, the model behaves in a predictable way, which proofs its correct implementation and physical relevance.

\section{ACKNOWLEDGEMENTS}

The authors are deeply thankful to Beata Ziaja and Viktor Tkachenko for numerous enlightening and motivating discussions. Partial financial support from the Czech Ministry of Education (Grants LG15013 and LM2015083) is acknowledged by N. Medvedev.

\section{REFERENCES}

[1] Schreiber, S., Faatz, B., "The free-electron laser FLASH," High Power Laser Science and Engineering 3, e20 (2015).

[2] Bostedt, C., Boutet, S., Fritz, D. M., Huang, Z., Lee, H. J., Lemke, H. T., Robert, A., Schlotter, W. F., Turner, J. J., et al., "Linac Coherent Light Source: The first five years," Reviews of Modern Physics 88(1), 15007, American Physical Society (2016).

[3] Pile, D., "X-rays: First light from SACLA," Nature Photonics 5(8), 456-457, Nature Publishing Group (2011).

[4] Beye, M., Sorgenfrei, F., Schlotter, W. F., Wurth, W., Föhlisch, A., "The liquid-liquid phase transition in silicon revealed by snapshots of valence electrons.," Proceedings of the National Academy of Sciences of the United States of America 107(39), 16772-16776 (2010).

[5] Beye, M., Wernet, P., Schüßler-Langeheine, C., Föhlisch, A., "Time resolved resonant inelastic X-ray scattering: A supreme tool to understand dynamics in solids and molecules," Journal of Electron Spectroscopy and Related Phenomena 188, 172-182 (2013).

[6] Medvedev, N., Rethfeld, B., "Transient dynamics of the electronic subsystem of semiconductors irradiated with an ultrashort vacuum ultraviolet laser pulse," New Journal of Physics 12(7), 73037 (2010).

[7] Ziaja, B., Wabnitz, H., Wang, F., Weckert, E., Möller, T., "Energetics, Ionization, and Expansion Dynamics of Atomic Clusters Irradiated with Short Intense Vacuum-Ultraviolet Pulses," Physical Review Letters 102(20), 205002 (2009).

[8] Hau-Riege, S. P., "Nonequilibrium electron dynamics in materials driven by high-intensity x-ray pulses," Physical Review E 87(5), 53102 (2013).

[9] Keski-Rahkonen, O., Krause, M. O., "Total and partial atomic-level widths," Atomic Data and Nuclear Data Tables 14(2), 139-146 (1974).

[10] Medvedev, N., Li, Z., Ziaja, B., "Thermal and nonthermal melting of silicon under femtosecond x-ray irradiation,” Physical Review B 91(5), 54113 (2015).

[11] Lorazo, P., Lewis, L., Meunier, M., "Thermodynamic pathways to melting, ablation, and solidification in absorbing solids under pulsed laser irradiation,” Physical Review B 73(13), 134108 (2006).

[12] Tkachenko, V., Medvedev, N., Li, Z., Piekarz, P., Ziaja, B., "Transient optical properties of semiconductors under femtosecond x-ray irradiation," Physical Review B 93(14), 144101, American Physical Society (2016).

[13] von der Linde, D., Sokolowski-Tinten, K., Bialkowski, J., "Laser-solid interaction in the femtosecond time regime," Applied Surface Science 109-110, 1-10 (1997).

[14] Medvedev, N., Jeschke, H. O., Ziaja, B., "Nonthermal phase transitions in semiconductors induced by a femtosecond extreme ultraviolet laser pulse," New Journal of Physics 15(1), 15016 (2013).

[15] Medvedev, N., Zastrau, U., Förster, E., Gericke, D. O., Rethfeld, B., "Short-Time Electron Dynamics in Aluminum Excited by Femtosecond Extreme Ultraviolet Radiation," Physical Review Letters 107(16), 165003 (2011).

[16] Ritchie, R. H., Howie, A., "Electron excitation and the optical potential in electron microscopy," Philosophical 
Magazine 36(2), 463-481, Taylor \& Francis (1977).

[17] Medvedev, N. A., Rymzhanov, R. A., Volkov, A. E., "Time-resolved electron kinetics in swift heavy ion irradiated solids," Journal of Physics D: Applied Physics 48(35), 355303 (2015).

[18] Rymzhanov, R. A., Medvedev, N. A., Volkov, A. E., "Effects of model approximations for electron, hole, and photon transport in swift heavy ion tracks," Nuclear Instruments and Methods in Physics Research Section B: Beam Interactions with Materials and Atoms 388, 41-52 (2016).

[19] Medvedev, N., Li, Z., Tkachenko, V., Ziaja, B., "Electron-ion coupling in semiconductors beyond Fermi's golden rule," Physical Review B 95(1), 14309, American Physical Society (2017).

[20] Chapman, D. A., Gericke, D. O., "Analysis of Thomson Scattering from Nonequilibrium Plasmas," Physical Review Letters 107(16), 165004, American Physical Society (2011).

[21] Ziaja, B., Medvedev, N., "Modelling ultrafast transitions within laser-irradiated solids," High Energy Density Physics 8(1), 18-29, Elsevier Ltd (2012).

[22] Medvedev, N., Li, Z., Ziaja, B., "Thermal and nonthermal melting of silicon under femtosecond x-ray irradiation,” Physical Review B 91(5), 54113 (2015).

[23] Medvedev, N., Li, Z., Ziaja, B., "Thermal and nonthermal melting of silicon exposed to femtosecond pulses of Xray irradiation," SPIE Optics + Optoelectronics, L. Juha, S. Bajt, and R. London, Eds., 95110I, International Society for Optics and Photonics (2015).

[24] Kwon, I., Biswas, R., Wang, C., Ho, K., Soukoulis, C., “Transferable tight-binding models for silicon,” Physical Review B 49(11), 7242-7250, American Physical Society (1994).

[25] Graves, J. S., Allen, R. E., "Response of GaAs to fast intense laser pulses," Physical Review B 58(20), 627-633 (1998).

[26] Harald O. Jeschke., "Theory for optically created nonequilibrium in covalent solids," Technical University of Berlin (2000).

[27] Parrinello, M., Rahman, A., "Crystal Structure and Pair Potentials: A Molecular-Dynamics Study," Physical Review Letters 45(14), 1196-1199, American Physical Society (1980).

[28] Verlet, L., "Computer 'Experiments' on Classical Fluids. I. Thermodynamical Properties of Lennard-Jones Molecules," Physical Review 159(1), 98-103 (1967).

[29] Rapaport, D. C., The Art of Molecular Dynamics Simulation, Cambridge University Press (2004).

[30] Trani, F., Cantele, G., Ninno, D., Iadonisi, G., "Tight-binding calculation of the optical absorption cross section of spherical and ellipsoidal silicon nanocrystals," Physical Review B 72(7), 75423 (2005).

[31] Koyama, T., Yumoto, H., Tono, K., Togashi, T., Inubushi, Y., Katayama, T., Kim, J., Matsuyama, S., Yabashi, M., et al., "Damage to inorganic materials illuminated by focused beam of x-ray free-electron laser radiation," SPIE conference proceedings, L. Juha, S. Bajt, and R. London, Eds., 951107, International Society for Optics and Photonics (2015).

[32] Sokolowski-Tinten, K., Bialkowski, J., von der Linde, D., "Ultrafast laser-induced order-disorder transitions in semiconductors," Physical Review B 51(20), 14186-14198, American Physical Society (1995). 